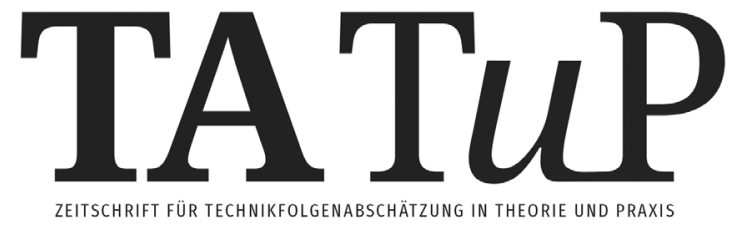

\section{Aus alt mach neu: Einblicke in den TATuP-Relaunch}

von Constanze Scherz, Jonas Moosmüller und Ulrich Riehm, Institut für Technikfolgenabschätzung und Systemanalyse (ITAS)

\section{Neue Qualität}

TATuP hat als wissenschaftliche Zeitschrift eine lange Tradition: Die Ausgabe 3/2016 schließt den 25. Jahrgang ab. Als „,TATuP - Zeitschrift für Technikfolgenabschätzung in Theorie und Praxis“ erscheint die erste Ausgabe nach dem Relaunch im August kommenden Jahres. In den vergangenen TATuP-Laboren haben wir verschiedene neue Rubriken und Designs getestet. Die Rückmeldungen der TATuP-Leserinnen und -Leser haben uns geholfen, den Blick für Bewährtes zu schärfen und zu erkennen, wo TATuP besser werden sollte.

Was haben wir vor? TATuP wird als wissenschaftliche Zeitschrift aufgewertet. Es wurden ein personelles Herausgebergremium und ein wissenschaftlicher Beirat etabliert. Wir werden am Konzept des interdisziplinären Themenschwerpunktes festhalten; neu ist, dass wir die Artikel dieser Rubrik doppelt begutachten lassen, mit dem Ziel, die Qualität der Artikel zu erhöhen und TATuP wissenschaftlich aufzuwerten sowie zügig als begutachtete Zeitschrift in internationalen Datenbanken (Scopus, ISI u.a.) zu etablieren. Auch Artikel, die nicht im Zusammenhang mit dem Themenschwerpunkt stehen, können für die Begutachtung eingereicht werden. TATuP ist und bleibt das zentrale Publikationsorgan für Beiträge aus dem breit verstandenen Feld der Technikfolgenabschätzung sowie angrenzender Forschungsgebiete (etwa Systemanalyse, Risikoforschung, Praktische Ethik, Forschung zur Nachhaltigen Entwicklung, Innovations- und Technikanalyse, Zukunftsforschung).

TATuP möchte gleichzeitig noch stärker als früher einen breiten, über die wissenschaftliche Community hinausgehenden Leserkreis anspre-

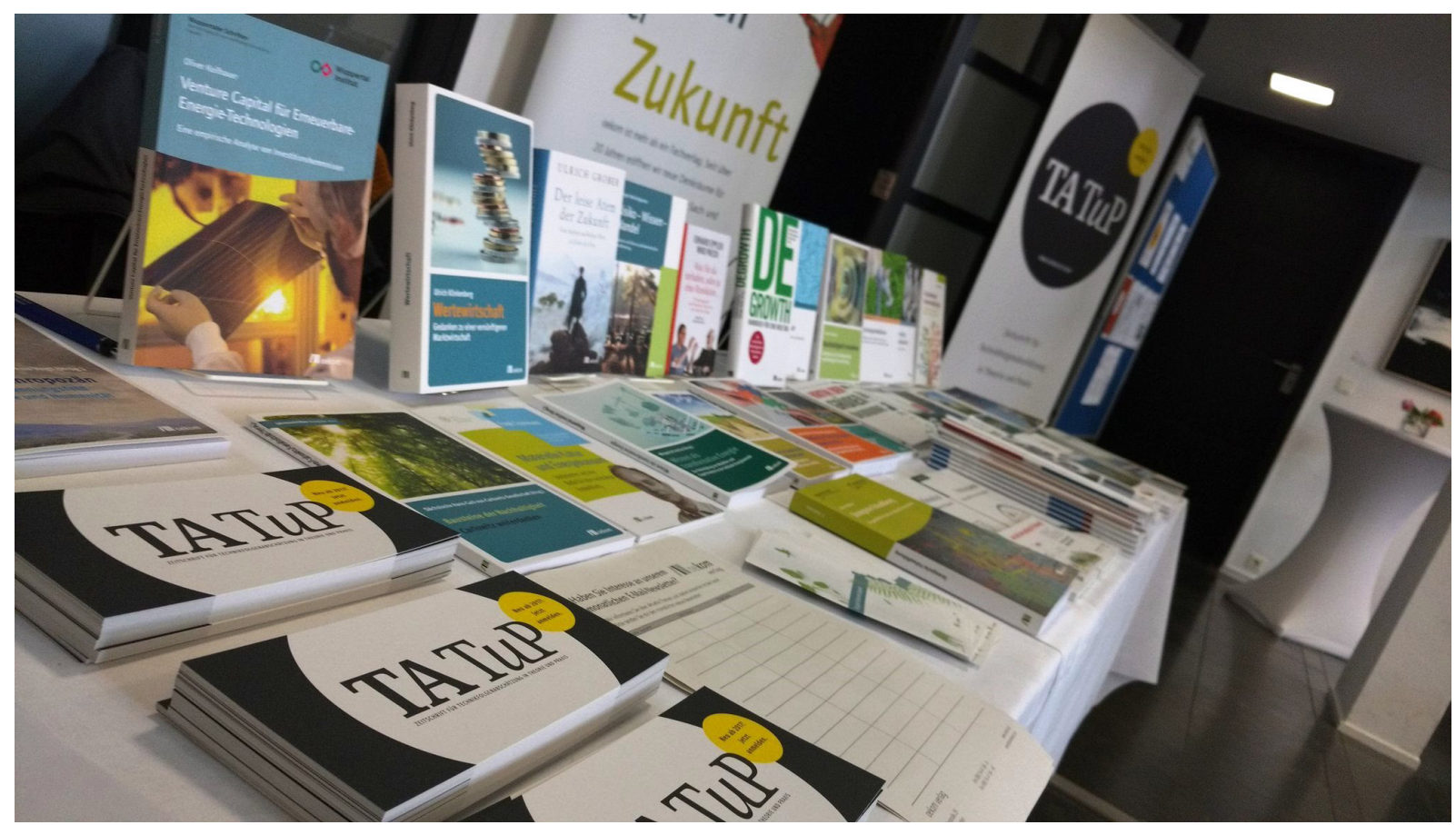

Bei Konzeption, Gestaltung und Vertrieb der neuen TATuP kooperiert die Redaktion mit dem oekom verlag. Hier der gemeinsame Stand auf der siebten Konferenz des Netzwerks TA (NTA7) im November 2016 in Bonn. 
chen. Eine News-Rubrik wird dazu Relevantes aus der TA-Landschaft präsentieren. Interviews, Rezensionen, Tagungsberichte werden reflektieren, was die TA-Community umtreibt. Alle, auch die wissenschaftlich begutachteten Artikel, sollen in ihrer Themen- und Sprachauswahl einen großen Leserkreis aus Wissenschaft, Politik, Wirtschaft und interessierter Öffentlichkeit ansprechen. TATuP bleibt sowohl in der Print- als auch in der Online-Ausgabe kostenfrei.

Mit diesem Anspruch startet die TATuP ins 26. Jahr ihres Bestehens. Die TATuP-Redaktion bleibt weiterhin am ITAS in Karlsruhe angesiedelt und kooperiert bei Produktion, Vertrieb und Marketing ab 2017 mit dem oekom verlag in München.

\section{Neue Offenheit}

TATuP wird ab 2017 als Open Access-Zeitschrift herausgegeben. Open Access bedeutet dabei mehr als freier Download von Artikeln von einem Server im Internet. Dies war bisher schon der Fall.

Legt man die „Berliner Erklärung über den offenen Zugang zum wissenschaftlichen Wis- sen" von 2003 zugrunde, die von Vertretern aller großen Forschungsorganisationen Deutschlands unterzeichnet wurde, dann bedeutet Open Access neben den freien, weltweiten Zugangsrechten, das Recht, diese Veröffentlichungen „,in jedem beliebigen digitalen Medium und für jeden verantwortbaren Zweck zu kopieren, zu nutzen, zu verbreiten, zu übertragen und öffentlich wiederzugeben sowie Bearbeitungen davon zu erstellen und zu verbreiten, sofern die Urheberschaft korrekt angegeben wird".

Dementsprechend werden die Artikel in TATuP mit einer modernen Creative Commons Lizenz CC BY 4.0 gekennzeichnet, die den Forderungen der „Berliner Erklärung“ entspricht. Die dadurch mögliche Nachnutzung - über Download, Ausdruck, Rezeption hinaus - mag für wissenschaftliche Publikationen auf den ersten Blick nicht von besonderer Relevanz sein. Denkt man aber z. B. an Nachdrucke in Sammelbänden, an die Bereitstellung digitaler Seminarapparate oder Übersetzungen, erschließt sich der Charme einer CC BY-Lizenz sofort - insbesondere für

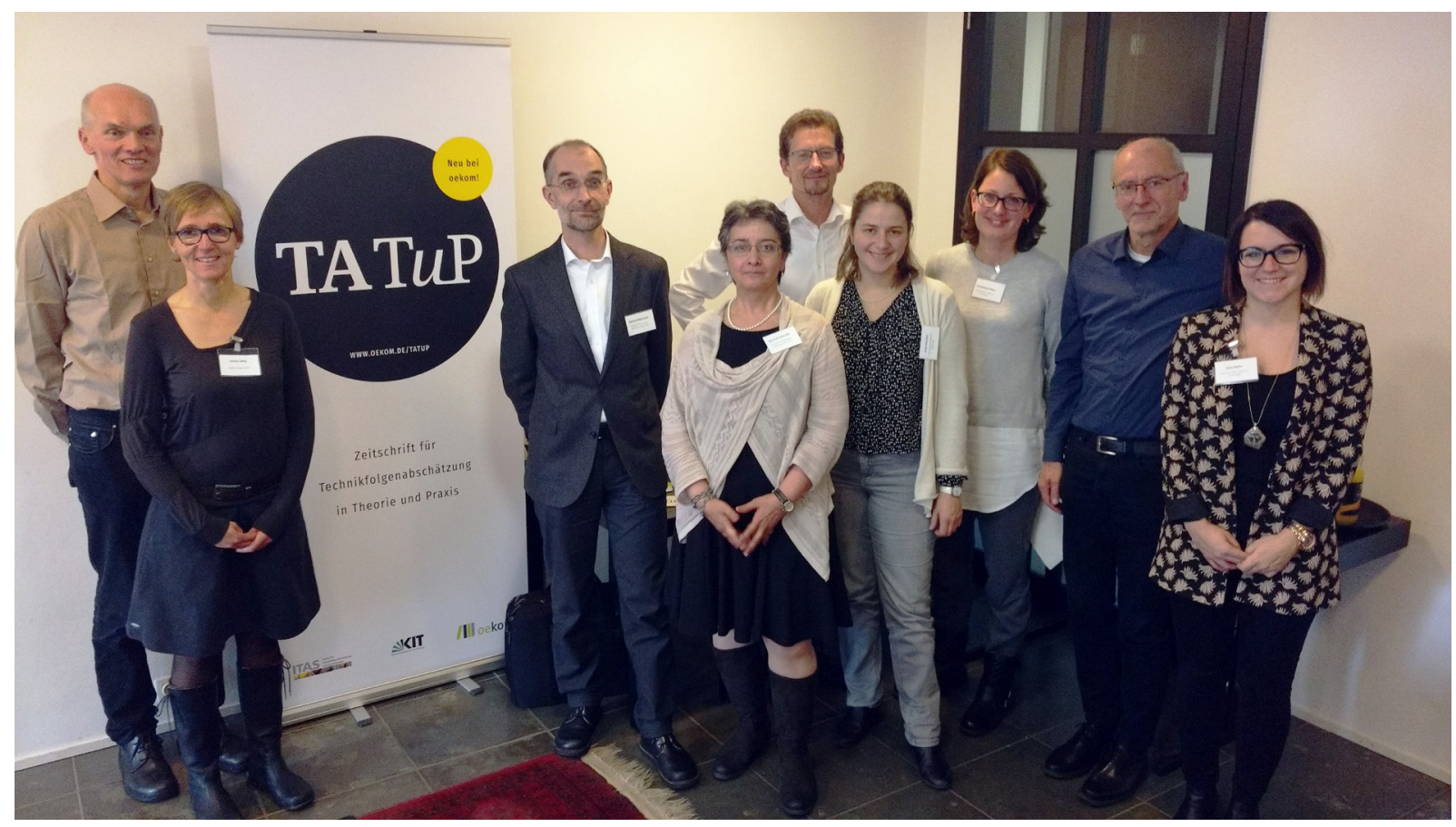

Erstes Treffen von Redaktion und neuem Herausgebergremium (v.l.n.r.): Ulrich Riehm (ITAS, Redaktion), Ulrike Sehy (oekom verlag), Michael Nentwich (ITA Wien, Herausgeber von Schwerpunktthema Heft 1-2/2017), Mahshid Sotoudeh (ITA, Herausgeberin), Stephan Lingner (EA European Academy, Herausgeber), Linda Nierling (ITAS, Herausgeberin), Constanze Scherz (ITAS, Redaktionsleitung), Armin Grunwald (ITAS, Vorsitzender Herausgeber) und Julia Hahn (ITAS, Redaktion). Nicht auf dem Bild: Regine Kollek (Universität Hamburg, Herausgeberin) und Marcel Weil (ITAS, Herausgeber) 
die nicht so seltenen Fälle, dass die Urheber nicht $\mathrm{zu}$ erreichen sind, weil sie z.B. nicht mehr wissenschaftlich tätig oder gar verstorben sind.

Für den Redaktions- und Begutachtungsprozess wird TATuP das weit verbreitete Open Journal System (OJS) einsetzen, das selbst als Open Source-Software frei verfügbar ist. OJS wird darüber hinaus unter der URL tatup.de ( $a b$ März 2017) das aktuelle Heft sowie das TATuPArchiv über eine nutzungsfreundliche Oberfläche zur Verfügung stellen. Reichhaltige Metadaten zu den Artikeln erlauben punktgenaue Recherchen, Normdaten, wie z. B. Autoren- oder Artikel-IDs (etwa ORCID oder DOI), bieten komfortable Verlinkungsmöglichkeiten, Standardschnittstellen, wie z. B. OAI-PMH, unterstützen den Datenaustausch und die Interoperabilität des Zugangs. Darüber hinaus wird man die Inhalte von TATuP in relevanten Nachweis- und Volltextdatenbanken wie Base, Google Scholar oder DOAJ finden. TATuP wird zudem auch über das Fachportal openTA des Netzwerks TA zugänglich sein.

\section{Neue Gestaltung}

Der TATuP-Relaunch ruht auf mehreren Säulen. Neben einem Begutachtungsverfahren, der konsequenten Open Access-Strategie und einem professionalisierten Vertrieb mit einem Verlagspartner spielt auch die grafische Gestaltung eine entscheidende Rolle, wenn es darum geht, Technikfolgenabschätzung hochwertig zu publizieren und in die gesellschaftliche Debatte einzubringen. Obwohl im Grundsatz ein wissenschaftliches Journal, öffnet sich TATuP explizit auch Leserinnen und Lesern außerhalb der wissenschaftlichen Community.

Daraus ergeben sich ganz besondere Anforderungen, die weit über einen klaren Stil und ein Höchstmaß an inhaltlicher Verständlichkeit hinausgehen: TATuP, so der Anspruch, braucht ein zeitgemäßes Erscheinungsbild, das allen Anforderungen an eine referierte wissenschaftliche Zeitschrift gerecht wird - angefangen bei konsequent zweisprachigen Abstracts und Überschriften, über ausführliche Autoreninformationen bis hin zu Keywords, DOIs und Lizenzangaben. Gleichzeitig gilt es, das Journal optisch so offen und ansprechend wie möglich zu gestalten, um Leserinnen und Leser zu erreichen, die sich der
TA aus einer nicht-wissenschaftlichen Perspektive nähern möchten.

Auf Basis dieser Vorüberlegungen arbeiten Verlag und Redaktion seit Herbst 2016 intensiv am neuen Layout. Einige Meilensteine sind bereits erreicht: Mit der Titelseite des neuen Hefts kann TATuP künftig eine „Visitenkarte“ mit großem Wiedererkennungswert vorlegen. Der Entwurf kombiniert einen „TATuP-Schriftzug“ mit einem von Ausgabe zu Ausgabe variierenden Kreiselement, der das jeweilige Thema der Ausgabe visuell unterstreicht.

Im Heftinneren erhalten die wissenschaftlichen Inhalte den nötigen Raum und angemessenes Gewicht. Immer wieder begegnen dem Leser aber auch einzelne Gestaltungselemente aus Magazinen: Ein- oder zweispaltige Fotografien, bebilderte Infoboxen zu Autoren oder als „pull quotes“ hervorgehobene zentrale $\mathrm{Zi}$ tate, die den Textzugang erleichtern. Auch TAFokus, der künftige Newsteil von TATuP, setzt neue Akzente: Neben klassischen Neuigkeiten zu Konferenzen, Calls und Projekten sorgen das regelmäßige Kurzinterview „5 Fragen an“, eine Kolumne zur N(T)Achrichtenlage oder eine TA-Grafik für eine informative und abwechslungsreiche Lektüre. Einblicke in die Arbeit am neuen TATuP-Design finden Sie online unter http://www.tatup-journal.de/entwuerfe.

Das neue Erscheinungsbild von TATuP bleibt bei alledem nicht auf das Printprodukt beschränkt, sondern findet seine Entsprechung auf den Online-Kanälen der Zeitschrift. Der künftige Webauftritt http://www.tatup.de greift (ab Frühjahr 2017) zentrale Gestaltungsmerkmale auf. Hinzu kommen entsprechende Präsenzen auf den Seiten des oekom Verlags (http://www. oekom.de/zeitschriften/tatup/) sowie auf Twitter (ab Frühjahr 2017) und Facebook (http://www. facebook.com/TAjournal).

\section{Neuer Bezug}

Bitte nutzen Sie die beiliegende Postkarte oder den Webauftritt des oekom verlags, um sich für den kostenfreien Bezug für TATuP und den TATuP-Newsletter anzumelden.

Die Redaktion freut sich insbesondere über Ihr Feedback und Ihre Anregungen für die „neue“ TATuP unter redaktion@tatup.de. 